\title{
An Atomic Force Microscopy Study of the Interactions Involving Polymers and Silane Networks.
}

\author{
Rodrigo L. Oréfice and Anthony Brennan.
}

\begin{abstract}
Silane coupling agents have been frequently used as interfacial agents in polymer composites to improve interfacial strength and resistance to fluid migration. Although the capability of these agents in improving properties and performance of composites has been reported, there are still many uncertainties regarding the processing-structure-property relationships and the mechanisms of coupling developed by silane agents. In this work, an Atomic Force Microscope (AFM) was used to measure interactions between polymers and silica substrates, where silane networks with a series of different structures were processed. The influence of the structure of silane networks on the interactions with polymers was studied and used to determine the mechanisms involved in the coupling phenomenon. The AFM results showed that phenomena such as chain penetration, entanglements, intersegment bonding, chain conformation in the vicinities of rigid surfaces were identified as being relevant for the overall processes of adhesion and adsorption of polymeric chains within a silane network. AFM adhesion curves showed that penetration of polymeric chains through a more open silane network can lead to higher levels of interactions between polymer and silane agents.
\end{abstract}

Keywords: Atomic force microscopy, silane coupling agents, interface.

\section{Introduction}

Silane coupling agents as interfacial modifiers in polymer composites

Although a rigid definition of "composites" has not been reached yet, they can be described as materials comprised of two or more constituents with very distinct composition, structure and properties separated by an interface. The aim in producing composites is to combine different materials in a single device with properties that cannot be obtained from the individual components. During the past 30 years, there has been a substantial development of composites for structural applications. The main support of this tendency is the possibility of producing composites with high mechanical properties and low density that can replace traditional materials such as steel and wood ${ }^{[1]}$.

One of the key elements of composites is the interface between reinforcing agents and polymer matrices. The study of interfaces in composites for structural applications is gaining much attention recently due to the recognition of their important role on the mechanical properties and environmental stability of the composites. In terms of mechanical properties, a rigid interface can lead to high levels of

Rodrigo Lambert Oréfice, Federal University of Minas Gerais (UFMG), Department of Metallurgical and Materials Engineering, LEPCom - Laboratory of Polymers and Composites Engineering, Rua Espírito Santo 35 - $2^{\circ}$ andar, 30160-030 Belo Horizonte, MG - Brazil, Phone: 55-31-238-1813, Fax: 55-31-238-1815, email: rorefice@demet.ufmg.br; Anthony Brennan, University of Florida, Department of Materials Science and Engineering, 32611 Gainesville, FL - USA. 
stress transfer from the matrix to the fibers. Environmental stability is another important major issue for advanced polymer composites. The interface between fibers (or fillers) and matrix can act as a pathway for corrosion that ultimately can lead to fibermatrix debonding, fiber pull-out and reduction of overall mechanical properties ${ }^{[2]}$. Although the understanding of the interfacial phenomena and the interface itself are crucial for the development of new composites with improved properties, the subject is clearly complex and far from being completely comprehended.

In order to improve interfacial properties of composites, surface modifications of the constituents are often performed. The goals in using these treatments are: (a) improve wetting by reducing differences in surface energy; (b) create possibilities of bond formation; (c) induce other type of interaction such as diffusion bonding or mechanical interlock; (d) improve resistance to fluid migration through the interface.

Methods of enhancing interactions between polymeric matrices and fibers/fillers have been tested and used, such as coupling agents, plasma, ozone treatments and polymer coatings or grafts ${ }^{[3]}$. The most traditional surface treatment that is used to control interfacial properties in composites, particularly in glass reinforced composites, is the use of silane coupling agents ${ }^{[4]}$.

Silane treatment on glass filers and fibers proved to be efficient in terms of improving mechanical strength of the composites ${ }^{[5]}$, elastic modulus ${ }^{[6]}$ and decreasing fracture toughness ${ }^{[6]}$ in relation to untreated fillers/fibers composites. The decrease in fracture toughness $\left(\mathrm{K}_{\mathrm{ic}}\right)$ can be explained by the fact that a weakly bonded interface can relieve crack energy.

Lifetime of composites was also enhanced by producing more rigid interphases in composites, such as those produced by using silane treatments ${ }^{[7]}$. The effect of water corrosion is particularly strong in weak interfaces and fiber debonding and pull-out are commonly seen when this type of interphase is present.

Silanes have the general form:

$\mathrm{R}_{\mathrm{n}} \mathrm{Si}(\mathrm{Y})_{(4-\mathrm{n})}, \quad$ where: $\mathrm{R}, \mathrm{Y}=$ organic radicals;

The "Y" group is usually an alkoxy or chlorine group that can be readily hydrolyzed, while the nonhydrolyzable " $\mathrm{R}$ " group is the one responsible for interacting with the polymer resin.

These silane agents undergo hydrolysis reactions and the formed hydroxyl groups can then react with inorganic surfaces or with themselves to create a network. The organic part can be polymerized within the matrix if these radicals have unsaturated bonds. Monoalkoxysilanes or monochlorosilanes can usually give rise to a monolayer, since polycondensation via $\mathrm{Si}-\mathrm{O}-\mathrm{Si}$ bond formation is restricted. On the other hand, trialkoxysilanes or trichlorosilanes can produce highly cross-linked multilayered structure, particularly when water not bound to the surface is supplied $^{[8]}$.

Amino silane agents are one of the most commonly used types of coupling agent, mainly because they can open the epoxide rings of epoxy resins and then be very useful as coupling agents in epoxy-glass composites ${ }^{[9-11]}$. Application of them on filler particles and fibers includes a variety of procedures going from the use of aqueous to anhydrous solutions, high to low concentrated solutions, room temperature to reflux and vapor deposition and wide range of $\mathrm{pH}$ 's. The structure of alkoxysilanes is known to be very sensitive to the conditions of processing. Temperature influences the rates of hydrolysis and condensation, while $\mathrm{pH}$ can alter the ratio of those rates. Concentration of silane agents in solution also changes the rates of the reactions but also changes viscosity. The manipulation of these variables will lead to materials with higher or lower levels of cross-link density and variety of packing efficiency of siloxane rings and chains.

In the case of amino silane agents, the thickness of the deposited layers and presence of only chemically bound species in contrast to both chemically and physically adsorbed species are affected by the initial processing conditions ${ }^{[9]}$. Washing the films with boiling solvent usually can eliminate the physical adsorbed silane molecules. The presence of the physically bonded species are suggested to either create a beneficial interphase with the polymeric matrix or degrade properties of the matrix by plasticizing it.

Time of flight secondary ion mass spectroscopy (ToF-SIMS) and XPS (x-ray photoelectron spectroscopy) showed that the type of amino silane solution affects the thickness of the deposited film ${ }^{[11]}$. Extraction with warm water $\left(50^{\circ} \mathrm{C}\right)$ led to a decrease in the content of nitrogen measured by XPS caused by the dissolution of adsorbed silane agents. Further extraction with hot water $\left(100^{\circ} \mathrm{C}\right)$ and hot toluene led to an extended decrease in nitrogen observed by XPS, due to degradation of the silane network. 
As commented above, silane agents have been applied in many systems throughout the last couple of decades using a series of different procedures of deposition. However, the effect of the structure of deposited layers (produced by the different methods of preparation) on the properties of the systems is poorly known. Moreover, the mechanisms involved on the interactions that occur when polymeric chains face silane networks are even more obscure. Another controversial point related to silane coupling agents in composites is whether or not the organic functionalities of the silane (organic groups other than the alkoxy groups) can actually bond to the polymeric matrices or they will only act as a compatibilizer for the surface energies. Although in some systems, primary bond formation between silane molecules and polymer had been already identified, in most of the cases, there are still some uncertainties about the real mechanisms of coupling ${ }^{[9]}$.

Interactions across interfaces measured by Atomic Force Microscopy (AFM)

Despite the fact that the importance of interfaces in a large variety of processes is well recognized, the structure of interfaces and surfaces is much less characterized than the bulk of materials. The main reasons for this lack of information are probably the combination of difficulties of searching the top layers of the material and also the usual and notorious unstable character of interfaces and surfaces.

Atomic Force Microscopy has rapidly spread throughout many fields of science due to its high versatility in using different types of physical and chemical aspects of surfaces and probes to give the desired output. More than allowing the processing of images on a nanometric scale, AFM has demonstrated to be very useful in investigating the magnitude of chemical and physical-chemical forces between materials on a molecular scale. Interactions between sharp AFM tips and samples can be monitored and the information obtained can be associated with physical-chemical and chemical processes, such as adhesion, van der Waals, electrostatic and even primary forces ${ }^{[12-14]}$.

The overall goal of this work is to test the hypothesis that Atomic Force Microscopy can serve as a tool to study surface and interfacial interactions in composites, by providing fundamental information in terms of the relationship between structure of the interfaces of polymer composites and properties of the system.

The specific goal of this work was to investigate the interactions between polymers and amino silane networks with different structures, deposited on silica, using AFM. The influence of the structure of the silane network on the interactions with polymers was studied. Fundamental questions about the coupling mechanism involved between silane agents and polymers in composites might then be answered by using the AFM as a tool to simulate the interface. In order to simulate the composite interface, polymers grafted on a tip will be forced against a silica surface treated with silane agents and interactions between them will be monitored. Chemical and physicalchemical interactions in polymer-silane network systems can then be analyzed.

\section{Experimental Procedure}

\section{Preparation of modified AFM tips and samples}

In this work, AFM was used to monitor interactions between a sulfonated polysulfone and amino silane networks with different structures deposited on silica surfaces. Modified AFM tips were produced by attaching glass spheres to AFM cantilevers. The glass spheres were also modified by grafting polymer on their surfaces. The polymer chosen was a sulfonated polysulfone (SPSF), because this polymer can readily interact with functional groups present in the amino silane networks, such as amine groups, through hydrogen bond formation, and eventually through the formation of primary sulfonamide bonds. This same procedure involving the modification of AFM tips with SPSF grafts was successfully employed in another work to study the interactions between polymers and inorganic surfaces ${ }^{[15]}$.

\section{Preparation of Sulfonated Polysulfone}

The sulfonation of polysulfone (PSF) was performed by dissolving a commercially available polysulfone (Udel ${ }^{\circledR}$ - Union Carbide) in dichloroethane $(10 \% \mathrm{wt})$. Chlorosulfonic acid was added to the solution at room temperature and the resultant mixture was stirred for 30 minutes. The sulfonation process was analyzed using NMR. Nuclear Magnetic Resonance (NMR) ${ }^{1} \mathrm{H}$ NMR $200 \mathrm{MHz}$ spectra were obtained with a Varian XL-Series NMR Super- 
conducting Spectrometer system. Tetramethyl silane was used as an internal reference material. Dimethylformamide- $d$ was used as solvent for the sulfonated PSF.

${ }^{1} \mathrm{H}$ NMR spectra of unmodified polysulfone and sulfonated polysulfone (SPSF) are depicted in Figure 1 . Only the portion of the spectra related to the aromatic portion of polysulfone is shown in this figure since usually sulfonation proceeds by substitution of protons on the aromatic rings by sulfonic acid groups $\left(-\mathrm{SO}_{3} \mathrm{H}\right)$. Since each proton position or group of positions on benzene rings were subjected to different chemical environments in PSF, distinct chemical shifts were observed for protons situated in different positions in the spectrum of polysulfone. Protons closer to the ether linkage had the largest upfield shift. The letters on the peaks in Figure 1 are related to each proton position on the benzene ring. The $c$ and $d$ protons are located on the rings of the diphenyl sulfone unit, while the $a$ and $b$ protons are found on the rings of the Bisphenol A unit, as also illustrated in Figure 1. The NMR spectrum of the SPSF is also illustrated in Figure 1. Peaks due to the protons named $c$ and $d$ in the sulfonated polysulfone were observed at same locations and magnitude (number of protons involved) as in the spectrum of unmodified polysulfone. Thus, substitution took place preferably on the Bisphenol A rings, as theorized. The four $\mathrm{a}$ and $\mathrm{b}$ protons on the substituted ring are nonidentical and are labeled $a^{\prime}$ and $b^{\prime}$, for those on the opposite side of the sulfonic acid group and $e$ for the proton adjacent to the new introduced group. Thus, the positions of $a$ and $b$ protons on the unmodified ring were essentially unchanged when compared to the original polysulfone and new peaks were observed due to the presence of new $a^{\prime}, b^{\prime}$ and $e$ protons. The substitution should have taken place preferentially on the ortho position in relation to the ether group.

The degree of substitution can be calculated from NMR data by basically rationing the areas under the peaks associated with the chemical modification. For the NMR spectrum reproduced in Figure 1, the degree of substitution was equal to 0.5 .

\section{Preparation of AFM modified tips}

In order to simulate the interface proposed (polymer against silane networks), conventional AFM tips were modified by attaching glass spheres (Polyscience, $15 \mu \mathrm{m}$ radius) to AFM cantilevers. Before

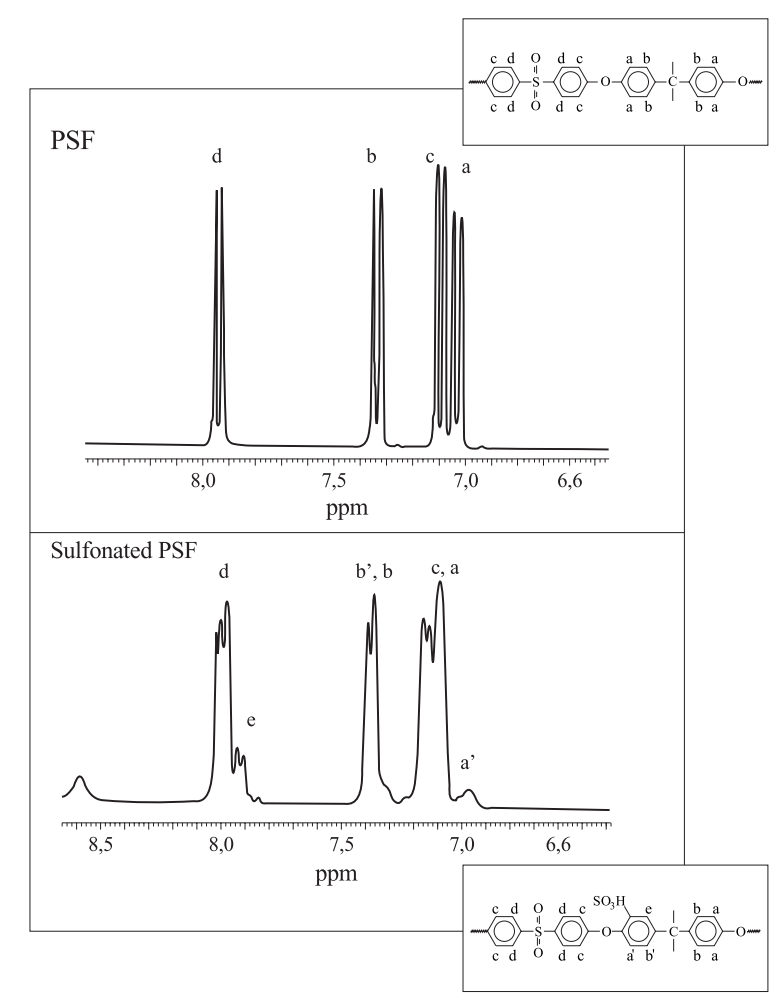

Figure 1. NMR spectra of polysulfone (PSF) and sulfonated polysulfone.

attaching them to cantilevers, SPSF was grafted onto the surface of the glass spheres. The sulfonated polymer used had a degree of substitution of 0.5 , according to NMR studies. In order to graft SPSF on spheres, their surfaces were firstly treated with aminopropyl triethoxysilane (APS) by dispersing the spheres in an ethanol-water-APS solution for one hour and curing them at $110{ }^{\circ} \mathrm{C}$ for 12 hours. The APS treated spheres were then reacted with SPSF by dispersing them in a SPSF-dimethylformamide solution for 1 hour. Sulfonamide bonds were processed by submitting the treated spheres to a heat treatment at $120{ }^{\circ} \mathrm{C}$ overnight under vacuum. Finally the grafted spheres were washed extensively with dimethylformamide, to remove any non-bonded polymer. The success of the grafting technique was confirmed with XPS results ${ }^{[15]}$.

The modified silica spheres were then attached onto AFM silicon nitride cantilevers. An epoxy resin was used to glue the sphere to the cantilever. The epoxy and sphere were transferred to the cantilever using a micromanipulator coupled with an optical microscope.

\section{Preparation of the substrates}

The substrates used for the deposition of amino silane were silicon wafers that had their surfaces oxidized at $420^{\circ} \mathrm{C}$ in air. After the oxidation process, 
the formed silica layer was hydrated by dipping the samples in boiling water.

Three different types of amino silane networks were deposited on silica substrates:

1) Substrates were dipped for one hour in toluene0.3 vol\% aminopropyl triethoxysilane (APS) solution. The procedure was performed in an environment with $15 \%$ relative humidity, so that minor amounts of water were introduced into the APS-toluene solution.

2) Substrates were dipped for one hour in an ethanol-5\% vol. water solution containing $1 \%$ in volume of APS.

3) Some of the substrates treated in the ethanolwater-APS solution were dipped into a boiling water for one hour.

After the deposition, the substrates were washed with ethanol and dried overnight at $110^{\circ} \mathrm{C}$.

\section{Measurements}

A Nanoscope III (Digital Inst., Santa Clara, CA) was used in contact mode. In order to avoid the formation of a thin liquid film on the substrate that usually adds a capillary force component to the measured forces by AFM, a fluid cell accessory provided also by the manufacturer was used. Deionized water $(\mathrm{pH}=5.0)$ was used during the measurements. Water is not a good solvent for the sulfonated polysulfone used (low degree of substitution), but will swell the polymer.

AFM force-distance measurements can be performed by applying to the piezoelectric a saw-tooth type of curve. In this case, instead of keeping the height or force constant, as usual when images are being processed in contact mode, the piezoelectric moves the sample against the tip in the vertical direction (z direction) in a cyclic way. In each cycle, the deflection of the cantilever is recorded as a function of the travel of the piezoelectric. When the tip is approaching the surface of the sample, an attractive force is usually present, represented by anyone of the type of chemical forces (electrostatic, van der Waals, etc.). A "jump" of the tip towards the sample surface occurs when the elastic constant of the cantilever cannot keep up with the gradient of forces acting on the tip. When an initially in-contact tip is released from the surface, another characteristic jump is produced in which the tip sticks to the surface and an extra force is required to unload the tip. The magnitude of this force can be related to the adhesion between the materials on the tip and sample. Adhesion, in this case, can be attributed to one or a combination of phenomena such as primary chemical bond formation, entanglements, secondary bond formation, among others. Many sites in each sample were used to obtain the force-displacement curves, thus ensuring reproducibility of results.

\section{Results and Discussion}

In Figure 2, a drawing representing the whole technique developed to measure interactions between surfaces and polymeric entities grafted on modified AFM tips (glass sphere with polymer grafts) is shown ${ }^{[15]}$. In this scenario, the polymer chains are being brought continuously in and out of contact with the surface. This cyclic mechanism mimics natural processes where polymeric chains hit the surface of substrates looking for positions with lower energy to adsorb. As shown in this figure, the use of SPSF with a low degree of substitution favors the formation of few linkages between the polymer and the surface of the glass sphere during tip preparation, thus allowing the presence of fairly large non-bonded chain lengths.

In a set of experiments, one specific tip with polymer grafts was used to search the surface of the

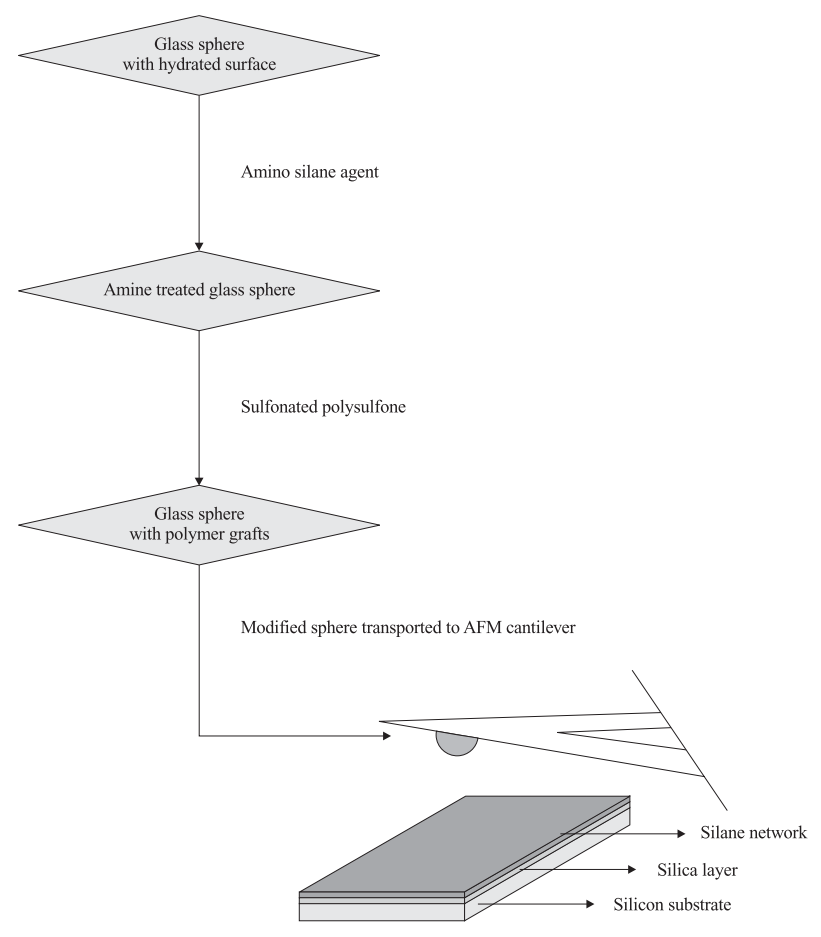

Figure 2. Procedure of AFM tip modification 


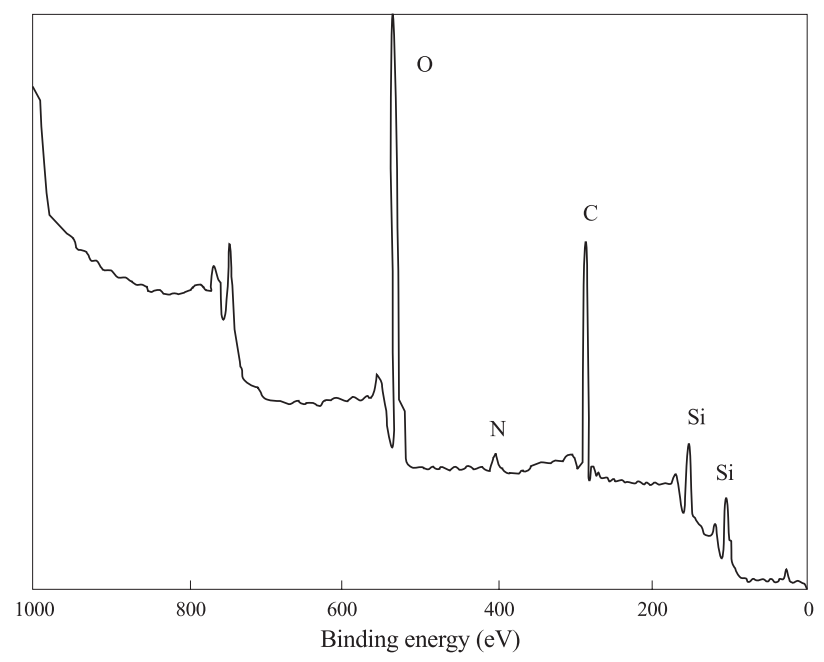

Figure 3. XPS spectrum of a silica surface treated with aminopropyl triethoxysilane

different substrates in all the measurements, and therefore it was possible to use the average value of the cantilever elastic constant (provided by the manufacturer, $\mathrm{k}=0.12 \mathrm{~N} / \mathrm{m}$ ) on the calculations (conversion of deflection of the cantilever in force). At the end of the measurements, the first sample investigated was again tested to ensure that no major degradation of the polymer grafts on the sphere had occurred during previous measurements.

Figure 3 shows a XPS (x-ray photoelectron spectroscopy) spectrum of the oxidized silicon substrate treated in an ethanol-water/APS solution. The presence of the nitrogen peak confirmed that the silane agent was successfully deposited on the surface of silicon wafers.

Table 1 reports the results of AFM measurements performed using the SPSF treated tip on the various APS treated silicon wafers. Values of distance and magnitude of forces associated with attractive minimum and adhesion are shown in this table. As observed in this table, attractive minimums were seen for all the samples regarding the interactions between APS treated surfaces and SPSF. Therefore, when the tip is approaching the surface of the samples, attractive

Table 1. Results from force-distance AFM curves using a SPSF tip and APS treated substrates.

\begin{tabular}{|c|c|c|c|c|}
\hline \multirow{2}{*}{$\begin{array}{l}\text { Procedure for } \\
\text { sample preparation }\end{array}$} & \multicolumn{2}{|c|}{$\begin{array}{c}\text { Extension - } \\
\text { attractive minimum }\end{array}$} & \multicolumn{2}{|c|}{ Retraction - adhesion } \\
\hline & $\begin{array}{l}\text { Distance } \\
(\mathbf{n m})\end{array}$ & $\begin{array}{l}\text { Force } \\
(\mathbf{m N} / \mathbf{m})\end{array}$ & $\begin{array}{c}\text { Distance } \\
(\mathbf{n m})\end{array}$ & $\begin{array}{l}\text { Force } \\
(\mathbf{m N} / \mathbf{m})\end{array}$ \\
\hline APSItoluene & 5.12 & 0.48 & 21.19 & 1.81 \\
\hline APSlethanol \water & 3.39 & 0.0153 & 10 & 0.564 \\
\hline APS boiled & 1.89 & 0.0055 & 11.87 & 0.118 \\
\hline
\end{tabular}

forces between the outermost parts of the grafted polymers and silane molecules (mainly the amine groups) forces the tip to jump to contact. In Figure 4 (top), a typical AFM extension curve for a modified tip-silane network system is shown.

In Figure 4, parts of the force-distance curves in which the tip is retracting from the surface are shown. As commented before, prior from being retrieved from the surface, the AFM tip was compressed against the substrate, allowing polymeric chains to fully interact with the surface. Therefore, retraction curves can potentially reveal phenomena such as chain penetration, entanglements, chemical bond formation, etc. In Figure 4, it is possible to observe that the value of adhesion is very dependent on the type of substrate used, i.e. dependent on the structure of the silane network. Higher values of adhesion were obtained for the sample treated with low concentration of APS in toluene. In this case, water was not introduced as a reactant, and should have been present in minor

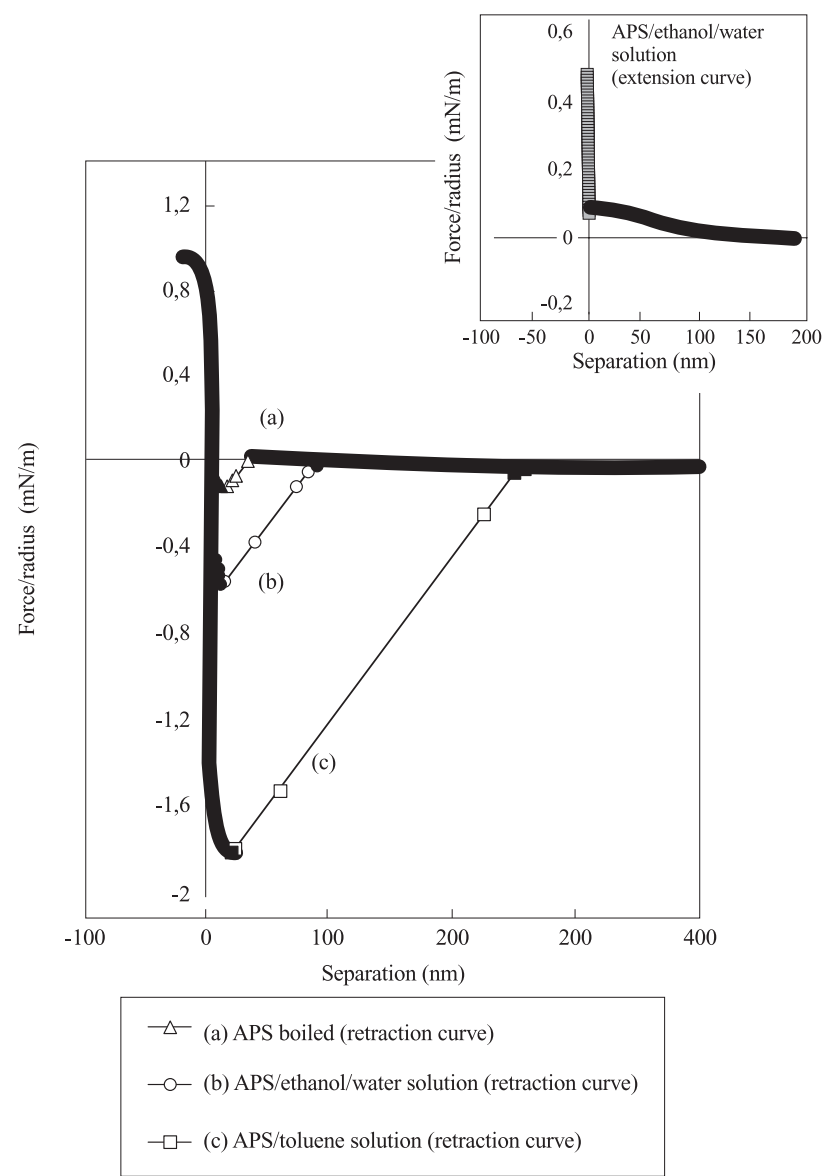

Figure 4. AFM force-distance curves for a sulfonated polysulfone modified tip against amine silane networks with different structures. On the top: typical extension curve for the substrates treated in an ethanol/water solution 
quantities only as a contaminant. Bonding between APS molecules and silanol groups $(-\mathrm{SiOH})$ on the surface of the silica layer, is in its majority processed via alcohol condensation (ethoxy groups reacting with hydroxy groups). Self condensation of the silane molecules via formation of siloxane bonds will be restricted and should occur for those silane molecules hydrolyzed by the minor amounts of water. The overall picture of the structure of silane networks deposited from the conditions described earlier should show the presence of an open network of polysiloxanes with low cross-link density. In this situation, penetration of polymeric chains within the loosely packed network is allowed in large extension, leading to a maximization of interaction between polymeric chains and polysiloxane chains via formation of hydrogen bonds between, for example, amine groups and sulfonic acid groups. Moreover, entanglements between polysiloxanes and organic chains can also occur. Thus, a combination of phenomena including interchain bonding and formation of entanglements is responsible for the high level of interaction (adhesion) observed on the analyzed system.

Lower levels of adhesion forces, in relation to the previous case, were measured when a tip with polymer grafts was brought in contact with an oxidized silicon wafer treated with APS in an ethanol-water solution. The amino silane networks deposited from the high APS concentrated ethanol-water solution should have a totally different network structure than the one produced by the deposition in a APS-toluene solution. Deposition of multilayers of a cross-linked network in this case is allowed due to the hydrolysis of the APS in solution and further self-condensation. Chemical and physically adsorbed entities should also be present due to hydrogen bonding between amine groups and silanol groups. This procedure used to obtain the deposited layers can lead to the formation of a very tight network with high cross-link density. This rigid network can restrict polymer chain interpenetration, that can reduce the number of entanglements between polysiloxane and polymer chains and also can lead to lower levels of bond formation between reactive groups on the chains.

As also shown in Figure 3 and Table I, the lowest level of adhesion was obtained for samples dipped into boiling water. This procedure can remove the adsorbed species as well as can degrade silane networks, leading to incomplete coverage ${ }^{[11,16]}$. In this case, the number of hydrogen bonds between reactive groups within the network and on the polymer, such as between amine groups and sulfonic acid groups is reduced due to defects on the network structure. Thus, it is clear that the concentration of reactive groups with high chemical affinity in both polymer and silane network is very important in determining the degree of interaction between the phases at the interface.

In terms of the properties of polymer composites, it can be proposed that higher levels of interfacial strength can be obtained by optimizing the structure of silane networks. Silane networks with lower crosslink densities should lead to improved properties by allowing interpenetration of chains and maximization of entanglements and chemical interactions between reactive groups. Presence of chemical affinities in both phases (polymer and silane) should also enhance interfacial properties by enabling higher levels of miscibility of the phases and higher degrees of interactions (through formation of chemical bonds) between them.

\section{Conclusions}

The experiments described and discussed in this work demonstrated that AFM can be used to study mechanisms that occur when polymer chains are present at the interface of composites. Basically, chain interpenetration, entanglements and intersegment bonding can be identified by AFM, which all are phenomena present at the interfaces of polymer composites.

In this work, the AFM technique was modified to simulate the interface of polymer composites. The effect of the structure of silane networks present at the surface of silica, i.e. cross-link density of the network, presence of reactive functional groups, and others, on phenomena such as chain entanglement, chain interpenetration and intersegment bonding could be studied using AFM. It was demonstrated here that the structure of silane networks can alter dramatically the type and magnitude of interactions between polymer chains and silane networks.

It was observed that adhesion between polymer and silane network can decrease if a highly crosslinked network structure is formed from the selfpolycondensation of silane agents, since this rigid structure can depress the penetration of the polymer chains into the network, restricting the formation of entanglements and interactions between silane 
functional groups and polymer. It was also demonstrated that the presence and concentration of chemical affinities in both phases (polymer and silane) is important in determining the degree of interactions between them.

The results of this work can be used to design high performance interfaces of composites by either employing polymers with specific architectures to interact at the interfaces or modifying the structure of the silane network to interact in an improved manner with the surrounding polymers (matrix).

\section{Acknowledgment}

The authors gratefully acknowledge the financial assistance of CNPq (Conselho Nacional de Desenvolvimento Científico e Tecnológico) of the Brazilian Government.

\section{References}

1. Mallick, P.K. - "Fiber-Reinforced Composites", Marcel Dekker, New York (1993).

2. Latour Jr., R.A.; Black, J. - J. Biomed. Mat. Res., 26, p.593-606 (1992).

3. Brennan, A.B.; Arnold, J.J.; Zamora, M.P. - J. Adhesion Sci. Tech., 8, p. 1031-48 (1995).

4. Jenneskens, L.W.; Schuurs, H.E.C.; Simmons, D.J.; Willems, L. - Composites, 25, p.504-11 (1994).

5. Liello, V.D.; Martuscelli, E.; Ragosta, G.; Zihlif, A. - J. Mat. Sci., 26, p.2100-6 (1991).

6. Amdouni, N.; Sautereau, H.; Gerard, J. F. - J. Appl. Polym. Sci., 46, p.1723-35 (1992).
7. Wagner, H.D.; Lustiger, A. - Composites, 25, p. 613-6 (1994).

8. Rothon, R. - "Particulate Filled Polymer Composites", Ed. Longman Scientific and Technical, London (1995).

9. Suzuki, N. and Ishida, H .- "A review on the structure and characterization techniques of silane/matrix interphases", Macromol. Sym., 108, p. 19-53 (1996).

10. Vandenberg, E.T.; Bertilsson, L.; Liedberg, B.; Uvdal, K.; Erlandsson, R.; Elwing, H.; Lundstroem, J. Colloid Interface Sci., 147, p.103-118 (1991).

11. Wang, D.; Jones, F.R. - Surface Interface Anal., 20, p.457-67 (1993).

12. Biggs, S. - "Steric and bridging forces between Surfaces bearing adsorbed polymer. An atomic force microscopy study”, Langmuir , 11, p.156162 (1995).

13. Grubmüller, H.; Heymann, B.; Tavan, P. - Science, 271, p. 997-9 (1996).

14. Rief, M.; Oesterhelt, F.; Heymann, B.; Gaub, H.E. - Science, 275, p.1295-7 (1997).

15. Oréfice, R.L.; Brennan, A.B.- "Evaluation of the interactions between polymeric chains and surfaces with different structures performed by an atomic force microscope". Submitted for publication in the Revista Materials Research.

16. Dupraz, A.M.P.; De Wijn, J.R.; Van der Meer, S.A.T.; Goedemoed, J.H. - J. Mat. Sci.: Mat. Medicine, 7, p.731-8 (1996).

Recebido: $30 / 07 / 98$

Aprovado: 30/10/98 